\title{
A Case of Recurrent Parasitic Fibroids Presenting as an Umbilical Mass
}

\author{
Jia Yi Lee* and Su Ling Yu \\ Department of Obstetrics and Gynecology, Singapore General Hospital, Singapore
}

*Corresponding author: Jia Yi Lee, Department of Obstetrics and Gynecology, Singapore General Hospital, Singapore.

Received Date: October 24, 2019

Published Date: October 29, 2019

\begin{abstract}
Fibroids are a common condition for women, and parasitic fibroids have been described to be rare complications following power morcellations during laparoscopic myomectomies. With improvement in surgical technique and training, laparoscopic myomectomies have become increasingly prevalent. Our case report of an unusual case of parasitic fibroids that recurred despite excision, highlights the difficulties in treating this complication. The atypical presentation of this case also reminds surgeons to be aware of the myriad of presentations that parasitic fibroids may have. Further research is still needed to determine the best approach of excision of parasitic fibroids.
\end{abstract}

\section{Background}

Uterine fibroids are a common condition affecting women worldwide. Treatment modalities are varied, with laparoscopic myomectomy first performed in 1979 [1]. Since the first successful laparoscopic myomectomy performed, it has now become an increasingly popular option due to the multiple advantages of a minimally invasive approach. A rare complication of parasitic fibroids as a result of laparoscopic morcellation during laparoscopic myomectomies was first described in 1997 [2], and multiple case reports have been published since, with the incidence estimated to be between 0.12 and $0.95 \%$ [3]. We describe the first case in our centre, of a patient who suffered recurrent parasitic fibroids after a single laparoscopic myomectomy was performed with morcellation and was required to undergo repeated excisions.

\section{Case Presentation}

A 42-year-old para 1 lady who delivered via a normal vaginal delivery, presented with a history of a mobile mass at the umbilicus region for 1 year. She had previously undergone an uncomplicated laparoscopic myomectomy 8 years ago for a single anterior wall fibroid measuring $9 \mathrm{~cm}$. The specimen was removed via power morcellation at the umbilical port site. She did not have any other medical or surgical history. Her periods were regular, normal in flow and she denied dysmenorrhea. Her vitals were stable, examination of the cardiovascular and respiratory systems was unremarkable.
On examination, her abdomen was soft, non-tender and nondistended. Previous laparoscopic surgical scars were seen. A mass measuring $4 \mathrm{~cm}$ was palpated at the umbilicus, it was mobile and firm in consistency. It was non tender, and no overlying skin changes were noted. On vaginal examination, her vulva, vagina, cervix appeared normal. Bimanual examination done elicited a uterus that was 8/52 in size, mobile and non-tender.

An ultrasound of the abdomen and pelvis was performed which revealed a $7.9 \times 6.7 \times 5.7 \mathrm{~cm}$ pedunculated fibroid in the posterior wall of the uterus. 3 discrete nodules ranging from $4.2 \mathrm{~cm}$ to $7.4 \mathrm{~cm}$ superior to the uterine fundus and 4 discrete nodules superficially subcutaneous tissue in the region of the umbilicus ranging from $1.4 \mathrm{~cm}$ to $5.2 \mathrm{~cm}$. They appeared hypoechoic with intrinsic vascularity with a similar appearance to the uterine fibroid. No bridging vessels with the uterus could be detected.

She underwent a laparotomy, myomectomy and excision of parasitic fibroids. Due to the multiple locations of the fibroids and large size, a laparoscopic approach was not recommended. Intraoperatively, 3 fibroids were seen in the sub rectal layer under the umbilicus measuring $7 \mathrm{~cm}, 4 \mathrm{~cm}$ and $3 \mathrm{~cm}$. There was 1 additional fibroid seen in the sub rectal layer in the suprapubic region measuring $2 \mathrm{~cm}$. There was also an $8 \mathrm{~cm}$ fibroid arising from 
the posterior peritoneal wall in the pouch of Douglas. The uterus was $8 / 52$ size with a $1 \mathrm{~cm}$ pedunculated fibroid on the posterior fundus.

The sub rectal fibroids were dissected free and the base clamped, divided and ligated. The large pedunculated fibroid arising from the pouch of Douglas was also clamped, divided and ligated. The fibroid of the uterus was also dissected free and excised from the uterus, completing the myomectomies. The uterus defect was then closed with vicryl 2-0, while the serosal surfaces were repaired with Prolene 3-0 sutures. A copious abdominal washout was done, and the abdomen was closed in layers.

The patient recovered uneventfully and was discharged on postoperative day 3. Her post-operative review in 1 month confirmed that her wound was well healed and there were no adverse effects felt post-surgery. Histology confirmed that all specimens removed were benign leiomyomas. 6 months later, the patient complained of pain and swelling at the umbilical region for 1 week. Purulent discharge was excreting from the umbilicus and she was admitted for a suspected diagnosis of an umbilical abscess. On examination, her vitals were stable, and she was afebrile. Her abdomen was soft and non-distended, a $5 \mathrm{~cm}$ mass was noted at the umbilicus with overlying erythema. Purulent discharge was noted, and the mass was tender. It was mobile and fluctuant. Pelvic examination was unremarkable, the uterus was normal in size, mobile and nontender. A CT scan of the abdomen and pelvis was performed. The CT scan revealed a lobulated soft tissue nodule measuring $5.8 \times 4.9 \mathrm{~cm}$ at the umbilical region in the subcutaneous plane with minimal intraabdominal extension. Anteriorly, it was flushed with the skin. A $1 \mathrm{~cm}$ focus of fluid density within the mass possibly contributed to the patient's symptoms of umbilical discharge. No other collections were seen. The uterus appeared bulky and nodular with small illdefined hypodensities within, likely due to fibroids. Both ovaries appeared normal. No enlarged intraabdominal or pelvic lymph nodes were seen. A trace amount of pelvic free fluid was likely physiological.

The patient was treated with a course of cloxacillin for 1 week with resolution of the pain and discharge seen. However, the umbilical mass was persistent and did not resolve. She was counselled of the likely diagnosis of a recurrence of the umbilical parasitic fibroid and was agreeable to undergo repeat excision of the umbilical fibroid.

Intraoperatively, there was a $5 \mathrm{~cm}$ umbilical fibroid seen just below the subcutaneous tissues, but above the rectus. The fibroid was excised piecemeal and the defect was closed with vicryl. The underlying uterus and ovaries appeared normal. The abdomen was washed out and closed in layers.

Post operatively, the patient recovered well and was discharged uneventfully. Clinically, she did not experience any further recurrence of the umbilical mass or discharge at the 12 months follow up. Histology of the removed specimen confirmed benign leiomyomatas, consistent with the pre-operative diagnosis of a recurrent parasitic umbilical fibroid.

\section{Discussion}

It has been hypothesized that parasitic fibroids may be a complication that arises when there is accidental seeding that happens during power morcellation, usually done to remove the specimen during a laparoscopic myomectomy. The most common presentation is usually abdominal pain or abdominal distension, although up to $21.7 \%$ of women may be asymptomatic [3]. Our patient had a history of laparoscopic myomectomy with power morcellation, which increased the clinical suspicion for a diagnosis of parasitic fibroids, although this is certainly an uncommon diagnosis given the overall rarity of the condition. Fortunately for our patient, the location of the parasitic fibroid being in the sub rectal layer in our patient meant that the mass was superficial and thus was easily palpable and detected.

Treatment options involve surgical resection, either via a laparotomy, or a laparoscopy [4]. Although a minimally invasive approach is favoured for its fast recovery, the possibility of an occult sarcoma must be counselled for, as it has been shown to lower survival rates [5]. In our patient, the location of the mass being superficial and not in the abdominal cavity warranted a laparotomy instead of a laparoscopic approach. As parasitic fibroids may be found to be seeded on various locations in the abdomen, a multidisciplinary approach must be considered especially if it is suspected to be near other organs, making excision tricky [6].

Although the ideal technique and device have not been determined to date, confined morcellation is a method of eliminating the chance of tissue dissemination inside the abdominal cavity, in order to reduce the chance of parasitic fibroids during laparoscopic myomectomies. However, in our patient, there was minimal chance of tissue dissemination as a laparotomy was performed during the first recurrence. Despite this, another recurrence had occurred, therefore there may be other factors involved that affect the risk of recurrence. More studies would be useful to evaluate the risk factors for recurrence.

The risk of recurrence after excision of parasitic fibroids has not been determined so far, and this was the first case of recurrent parasitic fibroids reported in our centre. Patients should always be counselled of the possibility of recurrence before surgery is undertaken. The median diagnosis interval from laparoscopic myomectomies is 48 months [3,6], thus an adequate length of follow up after laparoscopic myomectomies should be considered.

This case is unique in its recurrent presentation of parasitic fibroids, highlighting the need for good counselling before and after surgery that recurrence is a possibility despite its rarity. In view of the lack of good evidence with regards to the best surgical method for excision, we recommend an individualized approach to determine the best surgical options for patients with parasitic fibroids.

\section{Acknowledgement}

None. 


\section{Conflict of Interest}

Authors declare no conflict of interest.

\section{Refernces}

1. Verkauf BS (1992) Myomectomy for fertility enhancement and preservation. Fertil Steril 58(1): 1-15.

2. Ostrzenski A (1997) Uterine leiomyoma particle growing in an abdominal-wall incision after laparoscopic retrieval. Obstet Gynecol 89(5 Pt 2): 853-854.

3. Van der Meulen JF, Pijnenborg JM, Boomsma CM, Verberg MF, Geomini PM, et al. (2016) Parasitic myoma after laparoscopic morcellation: a systematic review of the literature. BJOG 123(1): 69-75.
4. Salih AM, Kakamad FH, A H D, J Habibullah I, M Rauf G, et al. (2017) Parasitic leiomyoma: a case report with literature review. Int J Surg Case Rep 41:33-35.

5. Wong M, De Wilde RL, Isaacson K (2018) Reducing the spread of occult uterine sarcoma at the time of minimally invasive gynecologic surgery. Arch Gynecol Obstet 297(2): 285-293.

6. Lete I, González J, Ugarte L, Barbadillo N, Lapuente O, et al. (2016) Parasitic leiomyomas: a systematic review. Eur J Obstet Gynecol Reprod Biol 203:250-259. 